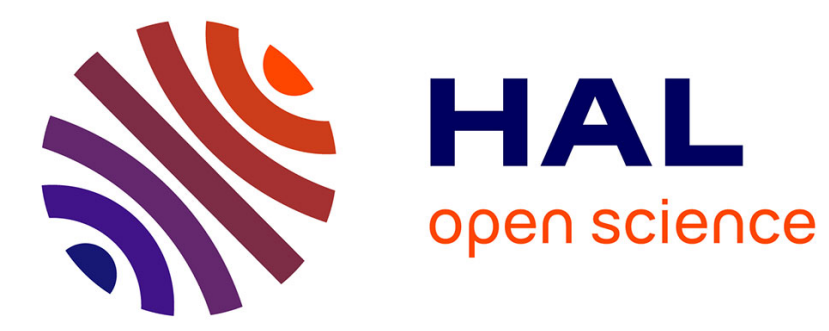

\title{
On the scattering properties of polyelectrolyte gels
} Jean-Louis Barrat, Jean-François Joanny, Phil Pincus

\section{To cite this version:}

Jean-Louis Barrat, Jean-François Joanny, Phil Pincus. On the scattering properties of polyelectrolyte gels. Journal de Physique II, 1992, 2 (8), pp.1531-1544. 10.1051/jp2:1992219 • jpa-00247750

\section{HAL Id: jpa-00247750 https://hal.science/jpa-00247750}

Submitted on 1 Jan 1992

HAL is a multi-disciplinary open access archive for the deposit and dissemination of scientific research documents, whether they are published or not. The documents may come from teaching and research institutions in France or abroad, or from public or private research centers.
L'archive ouverte pluridisciplinaire HAL, est destinée au dépôt et à la diffusion de documents scientifiques de niveau recherche, publiés ou non, émanant des établissements d'enseignement et de recherche français ou étrangers, des laboratoires publics ou privés. 
Classification

Physics Abstracts

$87.20 \mathrm{G}-61.41$

\title{
On the scattering properties of polyelectrolyte gels
}

\author{
Jean-Louis Barrat $\left({ }^{1}\right)$, Jean-François Joanny $\left({ }^{2}\right)$ and Phil Pincus $\left({ }^{3}\right)$ \\ ( $\left.{ }^{1}\right)$ Laboratoire de Physique, Ecole Normale Supérieure de Lyon, 46 Allée d'Italie, 69007 Lyon, \\ France \\ (2) Institut Charles Sadron, 5 rue Boussingault, Strasbourg, France \\ ( $\left.{ }^{3}\right)$ Department of Chemical and Nuclear Engineering and Materials Department, University of \\ California, Santa Barbara, CA 93106, U.S.A.
}

(Received 12 March 1992, accepted 15 May 1992)

Résumé . - Nous présentons un modèle simple pour étudier la diffusion de rayonnement par des gels polylectrolytes à l'équilibre de gonflement. Dans la limite d'écrantage faible où la longueur d'écran de Debye-Hückel est plus grande que la maille du gel, les interactions électrostatiques directes sont négligeables et le gonflement est dû à la pression osmotique des contreions. La tension créée par cette pression est transmise par les noeuds du gel aux chaines élastiques qui se comportent comme des chaines isolées avec une force extérieure appliquée aux extrémités. Le facteur de structure du gel est la somme d'une composante gelée due aux hétérogénéités de concentration figées et d'une composante thermodynamique due aux fluctuations de concentration. la composante gelée a un maximum pour un vecteur d'onde de l'ordre de l'inverse de la maille du gel et la composante thermodynamique a un maximum pour un vecteur d'onde beaucoup plus grand de l'ordre de l'inverse du rayon transverse des chaines. A temps longs, le facteur de structure dynamique relaxe vers la composante gelée du facteur de structure statique. A faible vecteur d'onde, la relaxation est diffusive avec un coefficient de diffusion de l'ordre du coefficient de diffusion de Stokes des blobs de Pincus des chaines étirées. Le coefficient de diffusion est minimal pour un vecteur d'onde de l'ordre de l'inverse du rayon transverse des chaines.

\begin{abstract}
We present a simple model for scattering properties of polyelectrolyte gels at swelling equilibrium. In the weak screening limit where the Debye-Hückel screening length is larger than the mesh size of the gel, the direct electrostatic interactions are negligible and the swelling is driven by the osmotic pressure of the counterions. The tension created by this pressure is transmitted through the crosslinks to the elastic chai $s$ which behave as isolated chains with an applied force at their end points. The structure $f_{c}$-tor of the gel can be split into a frozen component due to the average concentration heterogeneities and a thermodynamic component due to concentration fluctuations. The frozen component has a peak at a wavevector of the order of the mesh size of the gel, the thermodynamic component has a peak at a higher wavevector of the order of the inverse transverse radius of the chains. At infinite times the dynamic structure factor relaxes towards the frozen component of the static structure factor. In the limit of small wavevectors the relaxation is diffusive with a diffusion constant equal to the Stokes diffusion constant of the Pincus blobs of the stretched chains. The diffusion constant shows a minimum at a wavevector of the order of the inverse transverse radius of the chains.
\end{abstract}




\section{Introduction.}

Charged polymer gels often show striking macroscopic properties. Their swelling degree in water can be as high as 1000 [1] and can be monitored by changing the ionic strength; this makes them good candidates as superadsorbing materials for many practical applications. From a more fundamental point of view however, the properties of polyelectrolyte solutions and gels are not very well understood and the elegant scaling theories that have been successfully developed for neutral polymer solutions are not easily extended to charged systems. This is due both to the long range character of the Coulomb interactions and to the effect of the counterions that insure the electroneutrality.

The equilibrium swelling of neutral polymer gels results from a competition between the excluded volume interactions between monomers which tend to swell the gel and the entropic elasticity of the chains connecting the crosslinks. If the gel is not too heterogeneous, the conformational properties of the elastic chains are essentially the same as those of isolated linear polymer chains of the same molecular weight. Many properties of a neutral polymer gel are thus identical to those of a polymer solution where the chains are just on the verge of overlapping, this property is often referred to as the $\mathrm{c}^{*}$ theorem [2].

The properties of polyelectrolyte gels at swelling equilibrium are qualitatively different from those of polyelectrolyte solutions at the same concentration because of the different role of the counterions. In polyelectrolyte solutions, the counterions are more or less uniformly distributed and contribute to the screening of the electrostatic interactions. At swelling equilibrium, polyelectrolyte gels are in contact with a reservoir of solvent but the counterions are confined inside the volume of the gel. They exert thus a strong osmotic pressure that tends to swell the gel. This role of the counterions in the swelling of polyelectrolytes has been recognized very early in the seminal work of Katchalsky and his coworkers [3,4] who described the swelling of polyelectrolyte gels as a balance between the elastic energy and the osmotic pressure of the counterions. In this paper we study theoretically the properties of polyelectrolyte gels at swelling equilibrium. We first discuss the relative role of the counterion osmotic pressure and of the direct electrostatic interactions. We then focus on the weak screening limit where the counterions play a dominant role and the direct electrostatic interactions can essentially be neglected. We discuss macroscopic quantities such as the shear modulus and more microscopic properties such as the static structure factor measured in radiation scattering experiments and the cooperative diffusion constant measured either by quasielastic light scattering or by studying the swelling kinetics.

The paper is organized as follows: in section 1, we present a simple theory for the equilibrium swelling of a polyelectrolyte gel in a theta solvent. The cases of a salt free solvent and of a finite concentration of added salt are considered. In section 2, we investigate the consequences for the static properties (shear modulus and static structure factor) of the simple microscopic picture of the gel that emerges from the results of section 1. Dynamical properties are discussed within the same context in section 3 . The paper ends with a short discussion and a generalization of the results to the good solvent case.

\section{Equilibrium swelling of a polyelectrolyte gel.}

The equilibrium swelling of polyelectrolyte gels has been the subject of numerous theoretical studies (see for example $[5,6]$ and Refs. therein). In this section, we restrict ourselves to a particularly simple case, namely a polyelectrolyte gel immersed in a theta solvent. The only interactions are therefore of coulombic origin. We first consider the hypothetical case of a 
totally salt-free solvent, then the effect of adding a finite concentration of salt to the solvent. While our treatment does not aim at any originality $[3,4]$, we hope by using the theta solvent condition to free ourselves from some of the complexities that sometimes obscured the physical content of earlier treatments.

2.1 SAlt-FreE Theta SOLVENT. - We consider a macroscopic polyelectrolyte gel in a saltfree solvent: the number of monomers between crosslinks (i.e. the chain length), the distance between crosslinks and the fraction of charged monomers (bearing a charge $-e$ ) are denoted by $N, R$ and $f$ respectively. We assume throughout that the gel is ideal and does not have any large heterogeneities, we thus ignore the polydispersity of the chains between crosslinks. We also assume that the charge fraction $f$ is smaller than the value at which Manning's condensation [7] takes place, so that the Debye-Hückel linear screening approximation is valid. The total number of chains in the sample is $N_{c}$; the chains are strongly stretched so that the volume of the gel is $N_{\mathrm{c}} R^{3}$. The gel is immersed in a large volume $V$ of solvent. Finally, the number of positively charged counter-ions is $Q=N_{\mathrm{c}} N f$.

In a first step, we assume that all the counterions are confined to the volume of the gel. This is necessary to preserve electroneutrality at a macroscopic level, and can be further justified using the method decribed in the appendix. Moreover, we also neglect the contribution to the free energy and the osmotic pressure arising from local charge fluctuations inside the gel. The equilibrium swelling of the gel is thus determined by the balance between the osmotic pressure of an ideal gas of counterions, $k_{\mathrm{B}} T f c$ and the elastic pressure due to the stretching of the chains, $\frac{c}{N} k_{\mathrm{B}} T \frac{R^{2}}{R_{0}^{2}}$. Here $c=N / R^{3}$ is the monomer concentration in the gel, and $R_{0}=N^{1 / 2} a$ is the end-to-end distance of an unstretched chain. This immediatly yields:

$$
R \sim N f^{1 / 2} a
$$

This result should be compared with the classical estimate for the size of a polyelectrolyte chain in a very dilute solution [8], $R \sim N f^{2 / 3}\left(\ell_{\mathrm{B}} a^{2}\right)^{1 / 3}$, where $\ell_{\mathrm{B}}=\frac{e^{2}}{4 \pi \epsilon_{0} \epsilon_{r} k_{\mathrm{B}} T}$ is the Bjerrum length. In both cases, the chains are strongly stretched, $R \sim N$. For the gel, however, this stretching does not explicitly depend on the strength of the coulombic interactions: those only serve to confine the counterions through the electroneutrality constraint. Note also that if the fraction of charged monomers $f$ is small, the chains are more extended in a gel than in a dilute solution. This is different from the prediction of the $c^{*}$ theorem which for neutral gels predicts the same stretching for isolated chains and for the chains of the gel [2]. Taking into account local charge fluctuations of the counterions within the framework of the DebyeHückel theory [9] (but still considering the polymer as a uniform background) does not alter our result: the contribution of charge fluctuations to the osmotic pressure is $-k_{\mathrm{B}} T \kappa^{3} /(24 \pi)$, where $\kappa=\left(4 \pi \ell_{\mathrm{B}} f c\right)^{1 / 2}$ is the appropriate inverse Debye screening length [6]. This term is easily shown to be much smaller than the "ideal gas" contribution $k_{\mathrm{B}} T f c$ when equation (1) is verified. It turns out that the contribution from fluctuations in the local monomer density also has a negligible contribution to the free energy. This effect, however, is more subtle, and its discussion is postponed to the last paragraph of this section.

The fact that the strength of the electrostatic interaction $\ell_{\mathrm{B}}$ does not appear in equation (1) may first appear as a rather surprising result: the only forces that act on the polyelectrolyte chains are coulombic, so that the stretching necessarily originates from the presence of a local electric field. Equation (1) was obtained under the assumption of an electrically neutral gel. Clearly, this assumption cannot be true everywhere if the gel is swollen. This apparent 
contradiction is resolved if we realize that our reasoning does not exclude the presence of a narrow nonneutral region localized at the boundary of the gel. The electric fields that cause the swelling are localized in this region, but the contribution of this region to the thermodynamics, and therefore to the energy balance yielding (1), can be neglected. Similar situations, where forces are localized in boundary regions that are neglected when writing the energy balance equations, are not uncommon in classical electrostatics [10]. The same conclusion was also reached by Pincus [11] in his study of grafted polyelectrolyte layers, and can be further justified on the basis of the numerical solutions of the Poisson-Boltzmann equation presented in the appendix.

2.2 THETA SOLVENT WITH ADDED SALT. - A finite concentration $n$ of monovalent salt is now added to the solvent. A Donnan equilibrium $[12,13]$ establishes, in which the electroneutrality constraint on the gel volume plays the role of a semi-permeable membrane keeping the $Q$ positive counterions from diffusing away. The salt concentration inside the gel, $n_{\text {in }}$, is therefore smaller than the nominal salt concentration $n$. If we assume $n$ to be larger than the counterion concentration $f c$ inside the gel (if the reverse inequality is true, the results of the previous paragraph obviously apply), we find by equating the chemical potential of the salt inside and outside the gel a difference in osmotic pressure:

$$
\Pi_{\text {in }}-\Pi_{\text {out }}=k_{\mathrm{B}} T\left(f c+n_{\text {in }}-n\right)=k_{\mathrm{B}} T c^{2} f^{2} /(4 n)
$$

This result is most easily obtained by using the ideal gas expressions, $\mu=2 k_{\mathrm{B}} T \ln (n)$ and $\Pi / k_{\mathrm{B}} T=2 n$, for the chemical potential and osmotic pressure of the salt respectively. Remarkably, equation (2) remains unchanged if the Debye-Hückel limiting laws $\mu=2 k_{\mathrm{B}} T \ln (n)-\frac{1}{2} \kappa \ell_{\mathrm{B}}$ and $\Pi / k_{\mathrm{B}} T=2 n-\kappa^{3} /(24 \pi)$ (with $\kappa^{2}=4 \pi \ell_{\mathrm{B}}(2 n)$ ) are used instead.

Balancing this osmotic pressure difference against the elastic restoring force we obtain [6]:

$$
R \sim N^{3 / 5}\left(\frac{f^{2} a^{2}}{2 n}\right)^{1 / 5}
$$

The degree of swelling is reduced compared to the salt-free case. Note, however, that as in the salt-free case, it does not depend on the strength of the coulombic interactions.

2.3 Electrostatic interactions AND MONOMER DENSITY FLUCTUATIONS. - In the two preceding sections, the charges on the polymer chains were considered as simply providing a rigid negative charge background to the positively charged counterions. This would be justified if both the monomers and the counterions were uniformly distributed throughout the gel. In general, however, both densities are nonuniform quantities, and their fluctuations or inhomogeneities contribute to the free energy of the system. We already included the contribution due to the thermal fluctuations of the ionic density when using the Debye-Hückel law for the osmotic pressure of the ions. The effect of this correction was found to be negligible in the salt-free case and strictly zero in the presence of salt. At the same level of approximation, however, we must consider the contribution from the inhomogeneities in the monomer density. These inhomogeneities arise as a result of the presence of fixed crosslinks. It can be shown that at the level of the Debye-Hückel approximation (linear response of the ionic density to the monomer charge inhomogeneity) the energy associated with an inhomogeneous monomer density $c_{\mathrm{p}}(\mathbf{r})$ is

$$
E_{\mathrm{inh}}=\frac{f^{2}}{2}\left\langle\int \mathrm{d} \mathbf{r} \mathrm{d} \mathbf{r}^{\prime}\left(c_{\mathrm{p}}(\mathbf{r})-c\right) v_{\mathrm{DH}}\left(\mathbf{r}-\mathbf{r}^{\prime}\right)\left(c_{\mathrm{p}}\left(\mathbf{r}^{\prime}\right)-c\right)\right\rangle
$$


where $v_{\mathrm{DH}}(r)$ is the Debye-Hückel screened potential:

$$
v_{\mathrm{DH}}(r)=k_{\mathrm{B}} T \frac{\ell_{\mathrm{B}}}{r} \exp (-\kappa r)
$$

and $\kappa^{2}=4 \pi \ell_{\mathrm{B}}\left(2 n_{\text {in }}+c\right)$.

Introducing the Fourier components $\delta c_{\mathrm{p}}(q)$ of $\delta c_{\mathrm{p}}(r)=c_{\mathrm{p}}(r)-c$ and the structure factor of the gel

$$
S(q)=\frac{1}{N N_{\mathrm{c}}}<\delta c_{\mathrm{p}}(q) \delta c_{\mathrm{p}}(-q)>
$$

the value of the inhomogeneity contribution to the free energy can be rewritten as

$$
E_{\mathrm{inh}} /\left(N N_{\mathrm{c}} k_{\mathrm{B}} T\right)=\frac{f^{2}}{2} \int \mathrm{dq} S(q) \frac{4 \pi \ell_{\mathrm{B}}}{q^{2}+\kappa^{2}}
$$

The properties of $S(q)$ will be discussed in the next section using a very simplified model. Two features of this function are already obvious from its definition: first, it must have a peak at $q^{*} \simeq \frac{1}{R}$, reflecting the network structure of the gel with a meshsize $R$. Second, $S\left(q^{*}\right)$ is at most of order $N f$, since the contributions from different chains having random orientations will add in an incoherent way. As the structure factor is strongly peaked, we can estimate the integral in equation (7) by approximating it with $S(q) \sim f N q^{*} \delta\left(q-q^{*}\right)$. In this way we ignore logarithmic prefactors; we obtain:

$$
E_{\mathrm{inh}} /\left(Q k_{\mathrm{B}} T\right) \simeq \frac{N f \ell_{\mathrm{B}}}{R} \frac{1}{\left(1+\kappa^{2} R^{2}\right)}
$$

Let us first consider the salt-free case: here $\kappa^{2}=4 \pi \ell_{\mathrm{B}} f c$. Inserting the result of equation (1), one can check that the contribution from (8) to the swelling pressure is negligible, which a posteriori justifies the derivation of equation (1).

When a concentration $n>c$ of salt is added, two cases must be considered. If $\kappa R<1$ (weak screening) the contribution of $E_{\mathrm{inh}}$ to the swelling pressure is smaller by a factor $(\kappa R)^{2}$ than that arising from the Donnan equilibrium, equation (2). The result of equation (3) is still valid. If $\kappa R>1$ (strong screening), both contributions become of the same order of magnitude. Therefore equation (3) still applies, but the numerical coefficient is modified compared to the previous case.

Although the scaling law (3) does not change, it must be realized that the two cases of strong and weak screening correspond to rather different physical situations. In the latter case, the swelling is caused only by the counterions osmotic pressure. As in the salt-free case discussed above, the action of the counterions can be explained by the existence of electric fields localized at the gel boundary. Therefore the gel can be described as a network of stretched Gaussian chains, a picture that we shall use in the next section. When $\kappa R>1$, the contributions to the gel free energy obtained from equation (4) and by integrating equation (2) can be added to yield a total "electrostatic free energy":

$$
F_{\text {electrostatic }}=\frac{f^{2}}{2}\left\langle\int \mathrm{d} \mathbf{r} \mathrm{d} \mathbf{r}^{\prime} c_{\mathrm{p}}(\mathbf{r}) v_{\mathrm{DH}}\left(\mathbf{r}-\mathbf{r}^{\prime}\right) c_{\mathrm{p}}\left(\mathbf{r}^{\prime}\right)\right\rangle
$$

The properties of the gel are then similar to those of a semidilute polyelectrolyte solution at the same concentration. Since the Debye-Hückel potential is short-ranged, in the limit of high ionic strength the free energy can be described as a usual Flory type excluded volume free energy 
with strength $\ell_{B} \kappa^{-2}$. The gel is then similar to an ordinary Flory gel. In an intermediate range of ionic strength, the electrostatic interactions can contribute to the persistence length of the chains [14].

\section{Static properties of the gel.}

3.1 MODELISATION OF THE MICROSCOPIC STRUCTURE. - From now on, we limit ourselves to the salt-free solvent case or to the case of added salt in the weak screening limit. In both cases, the arguments developed in the previous section show that the gel can be described as a set of stretched Gaussian chains of length $R$ given by equation (1) or (3) respectively. The picture that we adopt is therefore that of a random network of Gaussian chains connecting the crosslinking points which are separated by an average distance $R$. Each crosslink of this network is actually the averaged position of a real crosslink. Each chain is subject to a force F of typical magnitude $3 k_{\mathrm{B}} T \frac{R}{N a^{2}}$ and of random direction. This force, transmitted through the crosslinks, creates the elongation $R$. From the magnitude $F$ of the force we extract the typical "blob size" $\xi$, i.e. the size over which the force only weakly perturbs the usual Gaussian statistics, and the number of monomers in a blob, $g$ :

$$
\begin{aligned}
& \xi=\frac{k_{\mathrm{B}} T}{F} \\
& g=\xi^{2} a^{-2}
\end{aligned}
$$

In the absence of added salt $\xi \sim f^{-1 / 2}$ while at higher ionic strength, $\xi \sim N^{2 / 5}\left(n / f^{2}\right)^{1 / 5}$.

Finally, we also introduce the transverse radius $R_{\perp}$ of the chain, which characterizes the lateral extension of the chain in the direction perpendicular to that of the force. Since the chains are Gaussian, this transverse radius is not affected by the stretching in the direction of the force, and we can define it as being the usual radius of gyration:

$$
R_{\perp}^{2}=N a^{2} / 6
$$

3.2 Shear MOdulus. - As a first application of our simple model, we estimate the shear modulus $G$ of the polyelectrolyte gel. This can be done by assuming that an homogeneous deformation is applied to the crosslinking points network. Let $\gamma$ be the magnitude of the displacement gradient. The average increase in chain length caused by the deformation is of order $(\gamma R)^{2}$. The cost in elastic energy per unit volume is therefore:

$$
F_{\mathrm{el}}=k_{\mathrm{B}} T\left(\frac{c}{N}\right)\left(\frac{R^{2} \gamma^{2}}{N a^{2}}\right)
$$

from which we obtain the shear modulus:

$$
\begin{aligned}
G & =k_{\mathrm{B}} T\left(\frac{c}{N}\right)\left(\frac{R^{2}}{N a^{2}}\right) \\
& \sim k_{\mathrm{B}} T f c \quad \text { (salt free case) } \\
& \sim k_{\mathrm{B}} T f^{2} c^{2} \ell_{\mathrm{B}} \kappa^{-2} \quad \text { (with added salt) }
\end{aligned}
$$

The shear modulus scales thus as the osmotic pressure that swells the gel (Eq. (2)). 
3.3 STATIC STRUCTURE FACTOR. - In the model described above, the structure factor $S(q)$ of the gel, defined by equation (6), is the sum of two components: one of these components, which we will call the "frozen" component and denote by $S_{0}(q)$, reflects the existence of a non-zero average value of the density Fourier component $c_{\mathrm{p}}(q),\left\langle c_{\mathrm{p}}(q)\right\rangle$. This non zero value stems from the existence of the crosslinks that restrict the translational motion of the chains and from the stretching of the chains. The existence of this component has recently been discussed in the context of neutral gels [15]. The second component, or fluctuating part of $S(q)$, is $S_{f}(q)=\frac{1}{N N_{\mathrm{c}}}\left(<c_{\mathrm{p}}(q) c_{\mathrm{p}}(-q)>-<c_{\mathrm{p}}(q)><c_{\mathrm{p}}(-q)>\right)$

The general behaviour of $S_{0}(q)$ and $S_{f}(q)$ can be infered from rather simple considerations. In the $q \rightarrow 0$ limit, $<c_{\mathrm{p}}(q)>\rightarrow 0$, the gel appears uniform. This is due to the fact that we study an ideal gel, in which no heterogeneities at length scales larger than $R$ are present. Thus, in this limit $S_{f}(q)=S(q)$. Since total charge fluctuations must vanish in this macroscopic limit, $f S(0)$ must be equal to the small wavevector value of the counterions structure factor. This yields $S(0) \simeq 1 / f$ in the absence of salt, and (from Eq. (2)) $S(0) \simeq f^{2} n / c$ when salt is added. When on the other hand $q R>1$, different chains contribute (as a first approximation) independently to the structure factors $S_{f}$ and $S_{0}$. These quantities can thus be computed by evaluating the contribution of a single Gaussian chain with one end fixed and the other subject to a force $\mathbf{F}$. These contributions to $S(q)$ and $S$ are respectively given by:

$$
\frac{1}{N} \int_{0}^{N} \mathrm{~d} m \int_{0}^{N} \mathrm{~d} n<\exp i \mathbf{q} \cdot\left(\mathbf{R}_{n}-\mathbf{R}\right)>=N \operatorname{Re}\left[g_{\mathrm{D}}\left(R_{0}^{2}\left(|\mathbf{q}|^{2}+2 \mathbf{i F} \cdot \mathbf{q} / \mathbf{k}_{\mathrm{B}} \mathbf{T}\right)\right)\right]
$$

(where $g_{\mathrm{D}}$ is the Debye function) and by

$$
\frac{1}{N} \int_{0}^{N} \mathrm{~d} m \int_{0}^{N} \mathrm{~d} n<\exp i\left(\mathbf{q} \cdot \mathbf{R}_{n}\right)><\exp i\left(\mathbf{q} \cdot \mathbf{R}_{m}\right)>
$$

One then has to sum the contributions of all the chains, which amounts to take an average over a randomly oriented force $\mathbf{F}$. Performing these calculations (and replacing the Debye function by its Lorentzian approximation [16]) we finally obtain:

$$
\begin{gathered}
S(q)=\frac{2 N}{q R} \operatorname{Atan}\left(\frac{q R}{2\left(1+q^{2} R_{\perp}^{2} / 2\right)}\right) \\
S_{0}(q)=\frac{3 R_{\perp}^{2}}{q R a^{2}} \int_{-q R}^{q R} \mathrm{~d} u \frac{1-2 \cos (u) \exp \left(-q^{2} R_{\perp}^{2}\right)+\exp \left(-2 q^{2} R_{\perp}^{2}\right)}{\left(q R_{\perp}\right)^{4}+u^{2}}
\end{gathered}
$$

From these expressions we can extract the scaling behaviour of $S_{0}(q)$ and $S_{f}(q)$ in the region $q R>1$. There are three different characteristic lengthscales $R, R_{\perp}$ and $\xi$ in the problem, and we must thus distinguish between three different regions:

(i) $q>\xi^{-1}$

$$
\begin{aligned}
& S_{f}(q)=12(q a)^{-2} \\
& S_{0}(q)=3 \pi q^{-3} a^{-2} R^{-1}
\end{aligned}
$$

In this region, the fluctuating contribution is the same as for a usual Gaussian chain, and the frozen component is essentially negligible in comparison.

(ii) $\xi^{-1}>q>R_{\perp}^{-1}$

$$
\begin{aligned}
& S_{f}(q)=N \pi(q R)^{-1}=3 \pi \xi q^{-1} a^{-2} \\
& S_{0}(q)=3 \pi q^{-3} a^{-2} R^{-1}
\end{aligned}
$$


Here the instantaneous structure factor $S(q) \simeq S_{f}(q)$ is similar to the structure factor for rods of length $R$, and its frozen component is still negligible.

(iii) $R_{\perp}^{-1}>q>R^{-1}$

$$
\begin{aligned}
& S_{f}(q)=N \pi\left(q R_{\perp}\right)^{2} R^{-1}=N \pi q \xi / 2 \\
& S_{0}(q)=N \pi(q R)^{-1}=\pi \xi q^{-1} a^{-2}
\end{aligned}
$$

In this region, the structure factor is dominated by its frozen component $S_{0}$, and is similar to that of a rod of length $R$.

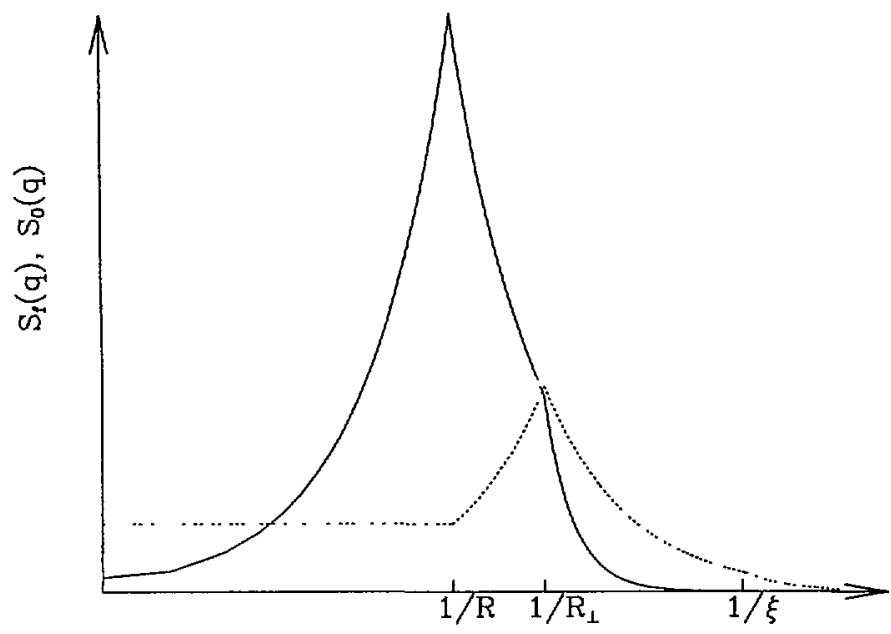

Fig. 1. - Schematic representation of the structure factors $S_{0}$ (full line) and $S_{f}$ (dotted line) for $N=100$ and $R=50 a$. The scale of the horizontal axis is logarithmic.

The general shape of $S_{0}(q)$ and $S_{f}(q)$ is sketched in figure 1. The behaviour of $S_{0}(q)$ in the region $q R<1$ could only be determined from a detailed knowledge of the crosslinking points network. Here we assumed a smooth crossover to the $q=0$ limit. In the same spirit, and since using formula (16c) for $q R=1$ yields (both with and without added salt) $S_{f}(1 / R)=\xi^{2} a^{-2}$ The structure factor crosses over smoothly to $S_{f}(0)$, it is thus natural to assume that $S_{f}$ is approximately constant in this region. The most remarkable feature of figure 1 is the existence of two peaks for $S_{0}$ and $S_{f}$ at very different positions, $q=1 / R$ and $q=1 / R_{\perp}$ respectively. The resulting $S(q)$, however, is peaked at $q=1 / R$ only as can be checked from (16a) and (16b). The height of the peak in $S_{0}(q)$ is $N$, while the peak in $S_{f}(q)$ has a magnitude $N^{1 / 2} \xi / a$.

These results can be given a simple physical interpretation: each chain is confined to a fixed cylinder of length $R$ and radius $R_{\perp}$. On a length scale larger than $R_{\perp}$, the fluctuations of the density inside this cylinder are irrelevant, and one gets for $S_{0}(q)$ the structure factor of a rod, equation (16c). The relevance of the fluctuations, however, increases as the length scale approaches $R_{\perp}$. This results in an increase of the fluctuating part $S_{f}$ for $q R_{\perp}<1$. When the length scale $1 / q$ becomes smaller than $R_{\perp}$, the chain still has instantaneously a rod-like configuration. This rod-like aspect, however, is smeared out by the transverse fluctuations as time evolves, and does not appear anymore in the frozen component of $S(q)$. Therefore $S_{f}$ now behaves as $1 / q$, and is much larger than $S_{0}$. Finally for $q$ larger than $\xi^{-1}$, we probe the correlations inside a blob which are by construction Gaussian. 


\section{Relaxation of concentration fluctuations.}

4.1 TWO-FLUIDS MODEL OF COLLECTIVE DIFFUSION. - In order to study collective diffusion in the gel in the long wavelength limit, we first use a simple "two-fluids model". The displacement field of the polymer network $u(\mathbf{r}, t)$ results from a mechanical balance between an elastic restoring force and a viscous drag force exerted by the solvent. The elastic force per unit volume can be written as $G \nabla^{2}(u(\mathbf{r}, t))$, where $G$ is an elastic constant which we take equal to the shear modulus calculated in the previous section. The drag force exerted by a solvent of viscosity $\eta$ flowing through a network with a meshsize $R$ is given by the Brinkman equation [17] and is of order $\eta R^{-2} \frac{\partial u(\mathbf{r}, t)}{\partial t}$ Balancing these two terms we obtain :

$$
G \nabla^{2}(u(\mathbf{r}, t))=\eta R^{-2} \frac{\partial u(\mathbf{r}, t)}{\partial t}
$$

and therefore a diffusion constant:

$$
D \sim \frac{G R^{2}}{\eta} \sim \frac{k_{B} T}{6 \pi \eta \xi}
$$

This diffusion constant is the same as that one would obtain by applying the Stokes-Einstein formula to a blob of size $\xi$. Note that $D$ is independent of $N$ in the salt-free case, $D \sim f^{1 / 2}$ and is a decreasing function of $N$ in the presence of salt, $D \sim N^{-2 / 5}\left(f^{2} / n\right)^{1 / 5}$

4.2 WAVEVECTOR DEPENDENT DIFFUSION CONSTANT. - The relaxation of density fluctuations at larger wavevectors, is measured through the time dependent structure factor:

$$
S(q, t)=\frac{1}{N N_{\mathrm{c}}}<c_{\mathrm{p}}(q, t) c_{\mathrm{p}}(q, 0)>
$$

The time dependent structure factor can be split into the frozen component $S_{0}(q)$ and a component $S_{f}(q, t)$ due to the thermal fluctuations which relaxes to zero at infinite time: $S(q, t)=S_{0}(q)+S_{f}(q, t)$. The initial relaxation rate $\Gamma(q)$ is then defined as the logarithmic derivative of $S_{f}(q, t)$ at time zero, and characterizes the short-time behaviour. From $\Gamma(q)$ we can extract a wavevector dependent diffusion coefficient:

$$
D(q)=\Gamma(q) / q^{2}
$$

The relaxation rate $\Gamma(q)$ can be obtained from the knowledge of the static structure factors $S_{0}$ and $S_{f}$ using the linear response formalism described in [16]. The final formula, which takes into account hydrodynamic interactions between monomers, reads:

$$
\Gamma(q)=\frac{k_{\mathrm{B}} T}{\eta} \int \frac{d^{3} \mathbf{k}}{(2 \pi)^{3}} \frac{S(\mathbf{k}+\mathbf{q})}{S_{f}(q)} \frac{\mathbf{k}^{2}-(\mathbf{k} \cdot \mathbf{q})^{2} / \mathbf{q}^{2}}{\mathbf{k}^{2}}
$$

This formula is similar to the one obtained for neutral polymer solutions [16]. One difference worth noting, however, is the presence of $S_{f}(q)$ rather than $S(q)$ in the denominator of equation (21). The origin of this difference is in the expression for the free energy associated with a deviation $\delta c_{p}(\mathbf{q})$ of the concentration from its average value. If we adopt the same Gaussian approximation used in [16], this energy reads in our case $k_{\mathrm{B}} T \frac{\delta c_{\mathrm{p}}(\mathbf{q}) \delta c_{\mathrm{p}}(-\mathbf{q})}{2 S_{f}(q)}$ The 
structure factor in the numerator of equation (21), on the other hand, results from averaging the hydrodynamic interactions over all monomer pairs, and involves therefore the full structure factor.

A detailed study of equation (21) using the structure factors computed in section 3 yields the following predictions:

$$
\frac{6 \pi \eta D(q)}{k_{\mathrm{B}} T}= \begin{cases}(6 \xi)^{-1} \ln (R / \xi) & \text { for } q<R^{-1} \\ (2 / \pi) R_{\perp}^{-2} q^{-1} \ln (1 / q \xi) & \text { for } R^{-1}<q<R_{\perp}^{-1} \\ (2 / \pi) q \ln (1 / q \xi) & \text { for } R_{\perp}^{-1}<q<\xi^{-1} \\ 3 \pi q / 8 & \text { for } \xi^{-1}<q\end{cases}
$$

In the small wavelength limit, we recover -except for a logarithmic correction- the prediction of the simple two-fluids model. The logarithmic factor is similar to the one that appears in the diffusion constant of rods with hydrodynamic interactions [16]. The diffusion constant $D(q)$ then becomes a decreasing function of $q$ in the range $R^{-1}<q<R_{\perp}^{-1}$, and reaches a minimum at $q \sim R_{\perp}^{-1}$. This minimum originates from the corresponding maximum in $S_{f}(q)$. At larger wavevectors, $\Gamma(q)$ displays a $q^{3}$ behaviour and is independent of the blob size $\xi$. A schematic representation of the wavevector dependence of $D(q)$ is given in figure 2 .

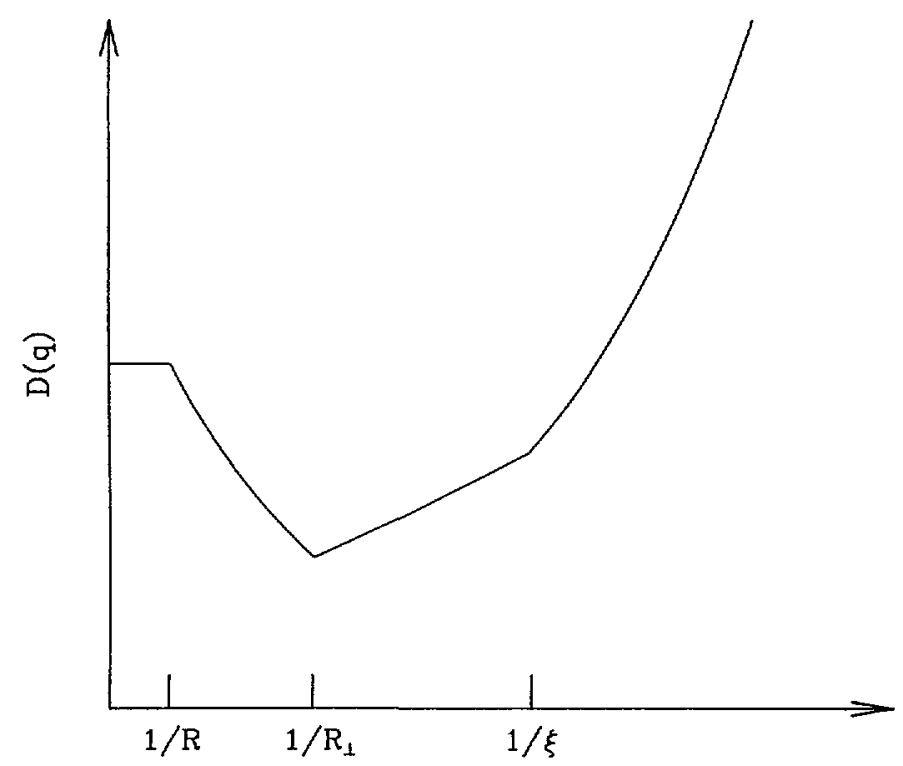

Fig. 2. - Schematic representation of the wavevector dependent collective diffusion coefficient $D(q)$. The scale of the horizontal axis is logarithmic.

An alternative way to study the relaxation dynamics on short length scales $(q R>1)$ is to compute the relaxation times of the Rouse modes of a stretched chain. These relaxation times 
were first obtained in [18]. The relaxation time $\tau_{p}$ of the $p$-th Rouse mode is given by:

$$
\tau_{p} \sim \begin{cases}\tau_{0} p^{-3 / 2} & \text { if } p>(\xi / a)^{2} \\ \tau_{0} p^{-2}(a / \xi) & \text { if } p<(\xi / a)^{2}\end{cases}
$$

where $\tau_{0}$ is a microscopic time and we ignore logarithmic factors. The blob size $\xi$ appears here as the length at which a crossover takes place from a Rouse free-draining behaviour at large length scales to a Zimm non-draining behaviour at short length scales. The relaxation times (23) can be inserted into the classical formula for the dynamical structure factor of a single chain [16]. The result, valid for $q R_{\perp}>1$ and for $t<\tau_{1}$, is

$$
\begin{aligned}
S(q, t) & =N^{-1} \int_{0}^{N} \mathrm{~d} n \int_{0}^{N} \mathrm{~d} m\left(\operatorname { e x p } \left[-\frac{(\mathbf{q}+2 i \mathbf{q} \cdot \mathbf{F})^{2}|n-m| a^{2}}{6}\right.\right. \\
& \left.\left.-\frac{N a^{2} q^{2}}{3 \pi^{2}} \int_{0}^{\infty} \frac{\mathrm{d} p}{p} \cos \left(\frac{p \pi|n-m|}{N}\right)\left(1-\exp \left(-t / \tau_{p}\right)\right)\right]\right\rangle_{\mathbf{F}}
\end{aligned}
$$

where the brackets denote an average over the orientation of the force $\mathbf{F}$. Taking the derivative with respect to $t$ at $t=0$, and dividing by $S_{f}(q)$, one recovers the result of equation (22) for $\xi^{-1}>q>R_{\perp}^{-1}$. This derivation makes clear that the $q^{3}$ dependence of $\Gamma(q)$ in this region, although similar to that of a Zimm chain [16], has a completely different physical origin. The dynamics is that of a Rouse free-draining chain, and the mobility $\left.\frac{\partial S(q, t)}{\partial t}\right|_{t=0}$ increases as $q^{2}$. The $q^{3}$ law is obtained when dividing by $S_{f}(q) \sim 1 / q$ to obtain the initial decay rate. For $q>\xi^{-1}$, on the other hand, one probes the correlations inside one blob, and the behaviour is that of a genuine Zimm non-draining chain.

Equation (24) also allows a study of the time dependence of $S_{f}(k, t)$ beyond the initial decay. Following [16] we find (for $\xi^{-1}>q>R_{\perp}^{-1}$ )

$$
S_{f}(q, t) \simeq S_{(q) \exp }\left(-2\left[\Gamma^{\prime}(q) t\right]^{1 / 2}\right)
$$

where

$$
\Gamma^{\prime}(q)=\tau_{0}^{-1}(q a)^{4} \xi a^{-1}
$$

The crossover from the single exponential decay to the stretched exponential decay (26) takes place for $t \sim \tau_{0} \xi q^{-2} a^{-3}$

\section{Conclusion.}

We have studied the properties of polyelectrolyte gels at swelling equilibrium. When the meshsize $R$ of the gel is smaller than the Debye-Hückel screening length, in the weak screening limit, we have shown in agreement with the classical theory that the swelling of the gel is driven by the osmotic pressure of the counterions. This pressure acts on the boundary of the gel and creates a tension transmitted to the internal chains through the crosslinks. The chains of the gel behave therefore as isolated chains stretched by an external force.

The macroscopic properties of the gel such as the $q=0$ limit of the structure factor are then dominated by the counterions. Both in the absence of added salt and at finite ionic strength, the shear modulus of the gel is of the order of translational osmotic pressure of the free ions that swell the gel. 
Due to the connectivity between the chains and the strong stretching of the chains we expect strong frozen microscopic heterogeneities of the gel concentration. The structure factor of the gel can be decomposed into a frozen component and a component due to thermodynamic fluctuations. The frozen component dominates in the intermediate range of wavevectors and has a strong peak at a wavevector of the order of the inverse of the meshsize of the gel. The thermodynamic contribution to the structure factor dominates at low wavevectors and has a peak at a larger wavector of the order of the inverse of the perpendicular radius of the chains. In a static scattering experiment the total structure factor is measured, and the signal should be dominated by the frozen component. The thermodynamic component relaxes with time and could be observed in a dynamic scattering experiment. We have discussed the relaxation in details in section 4 . The low $q$ behaviour could be probed by dynamic light scattering and the high $q$ behaviour using the neutron spin echo technique.

All these results are obtained for chains in a theta solvent where the only interactions are Coulombic. It has been recently emphasized however that interactions of non electrostatic origin can play an important role in polyelectrolyte problems. In a good solvent the same description in terms of stretched chains should apply. The non-linear elasticity of the chains can be treated using Pincus blob model [18]. The radius of the chains is then given by $R \sim N f^{2 / 5} a$ in the absence of salt and $R \sim N^{7 / 11}\left(\frac{f^{2} a^{2}}{2 n}\right)^{2 / 11}$ with added salt where the Flory approximation $\nu=3 / 5$ has been used for the swelling exponent. The results for the structure factor and the cooperative diffusion constant can be used in a good solvent whenever $q \xi<1$, the blob size being equal to $\xi \sim f^{-3 / 5}$ in the absence of salt and $\xi \sim N^{6 / 11}\left(n / f^{2}\right)^{3 / 11}$ at higher ionic strength. The case of a poor solvent is more subtle and deserves a more detailed analysis since the competition between the repulsive electrostatic interactions and the attrations can lead to a structuration of the solution $[19,20]$.

Finally, our model is limited to the weak screening limit and to gels at swelling equilibrium. In the strong screening regime, $\kappa R>1$, the swelling is driven by direct electrostatic interactions and the gel behaves as a semidilute polyelectrolyte solution. If the gel is not at swelling equilibrium but at a higher concentration $c$, the osmotic pressure of the free ions still dominates as soon as the radius $R=(N / c)^{1 / 3}$ is larger than the radius of an isolated chain given $R \sim$ $N f^{2 / 3}\left(\ell_{B} a^{2}\right)^{1 / 3}$ and the physics remain the same as for equilibrium gels; if the concentration is large enough so that the meshsize is smaller than this isolated chain value, electrostatic interactions become dominant and the gel also behaves as a semidilute solution.

\section{Acknowledgements.}

One of us (JFJ) would like to thank the Materials Department at UCSB for hospitality during the early stages of this work. Financial support from the Department of Energy (USA) under grant DE-FG03-87ER and from the Centre National de la Recherche Scientifique (France) is acknowledged.

\section{Appendix.}

In this appendix, we present a numerical solution of the Poisson-Boltzmann equation for a system made up of a negative charge $-Q e$ uniformly spread in a sphere of radius $L$ (the "gel") and of $Q$ monovalent positive counterions enclosed in a spherical cell of radius $d>L$. We are interested in checking the assumption made in section 2.1 that the counterions are confined to 
the region $r<L$, with a uniform density inside this region. Introducing the scaled variables:

$$
x=\frac{r}{R}, \tilde{\psi}=\frac{e \psi}{k_{\mathrm{B}} T}, \tilde{c}_{\mathrm{i}, \mathrm{p}}=\frac{c_{\mathrm{i}, \mathrm{p}} R^{3}}{Q}
$$

where $\psi$ is the electrostatic potential and $c_{i}, c_{p}$ are the charge densities of the ions and of the "gel" respectively, the Poisson Boltzmann equation can be written:

$$
\begin{aligned}
\frac{\mathrm{d}^{2}}{\mathrm{~d} x^{2}}(x \tilde{\psi}(x)) & =\frac{Q \ell_{\mathrm{B}}}{L}\left(\tilde{c}_{\mathrm{p}}(x)-\tilde{c}_{\mathrm{i}}(x)\right) \\
\tilde{c}_{\mathrm{i}}(x) & =\frac{\exp (-\tilde{\psi}(x))}{4 \pi \int_{0}^{d / L} \mathrm{~d} x^{\prime} x^{\prime 2} \exp \left(-\tilde{\psi}\left(x^{\prime}\right)\right)}
\end{aligned}
$$

and the boundary conditions are:

$$
\begin{array}{r}
\left.x \tilde{\psi}(x)\right|_{x=0}=0 \\
\frac{\mathrm{d}}{\mathrm{d} x}(x \tilde{\psi}(x))_{x=0}=0
\end{array}
$$

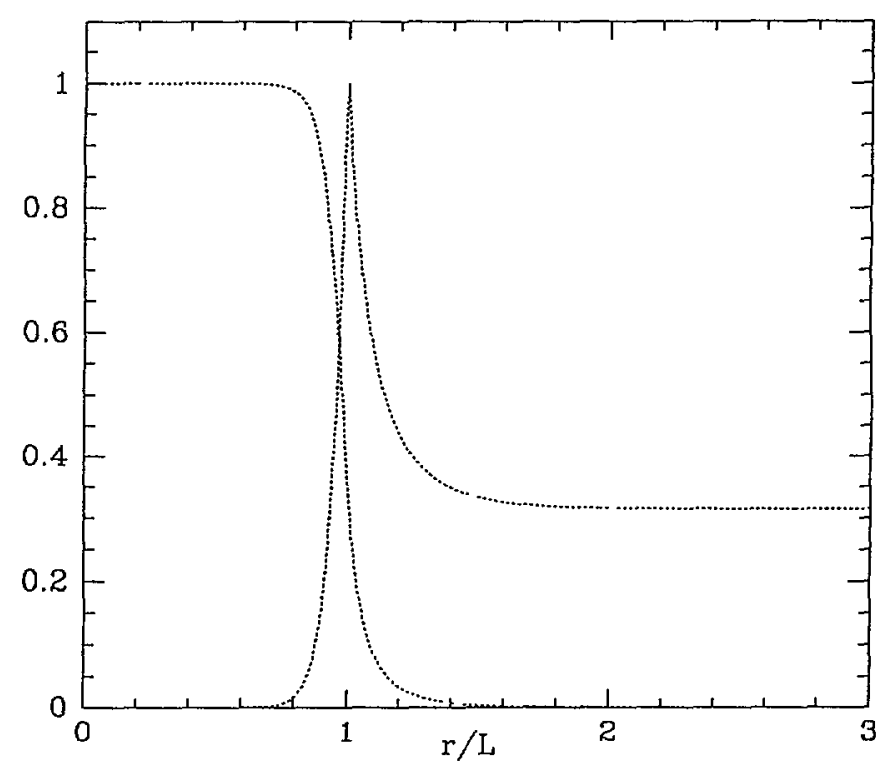

Fig. 3. - Numerical solution of the Poisson Boltzmann equation for a gel sample of radius $L$ inside a cell of radius $100 L . Q \ell_{\mathrm{B}} / R=1000$. Full line: normalized ionic concentration. Dotted line: electric field normalized by its maximum value.

For a given value of the two dimensionless parameters $Q \ell_{\mathrm{B}} / L$ and $d / L$, the problem defined by equations (A2) and (A3) can easily be solved iteratively, starting for example with 
a Gaussian distribution of counterions. We are interested here in the limit of a macroscopic sample, which means that the parameter $Q \ell_{B} / L$ is large, of order $N_{c}^{2 / 3} f^{1 / 2}$ if we accept the result of equation (1). The second parameter $d / L$ is the ratio of the container size to the gel size. In figure 3 , we present the numerical results obtained for $\tilde{c}_{\mathrm{i}}(x)$ when $Q \ell_{\mathrm{B}} / L=5000$ and $d / R=100$. The results for $d / R=1000$ or $d / R=10$ would be essentially undistinguishable from those presented here. As expected, we find that the counterions are localized inside the gel $(99.8 \%$ of them are found for $r<L)$, and that the electrostatic field inside the gel is 0 except at the boundary.

\section{References}

[1] Schosseler F., Mallo P., Cretenot C, Candau S.J., J, Dispersion Sci. Technol. 8 (1986) 321.

[2] de Gennes P.G., Scaling Concepts in Polymer Physics (Cornell University Press, 1985).

[3] Katchalsky A., Lifson S., Eisenberg H., J. Polym. Sci. A 7 (1951) 571.

[4] Katchalsky A., Michaeli I., J. Polym. Sci. A 15 (1955) 69.

[5] Flory P.J., Principles of Polymer Chemistry (Cornell University Press, 1954).

[6] Hill T.L., Introduction to Statistical Thermodynamics, Dover (1986).

[7] Manning G.S., J. Chem. Phys. 51 (1969) 954.

[8] de Gennes P.-G., Pincus P., Velasco R.M. and Brochard F., J. Phys. France 37 (1976) 1461.

[9] Landau L., Lifchitz E.M., Physique Statistique, sections 75 and 94, (Editions Mir, Moscou, 1967).

[10] Feynman R.P., Leighton R., Sands M., Lectures on Physics, (Addison-Wesley, 1964).

[11] Pincus P., Macromolecules 24 (1991) 2912.

[12] Donnan F., Z. Physik. Chem. A 168 (1934) 369.

[13] Donnan F., Guggenheim E., Z. Physik. Chem. A 122 (1932) 346.

[14] Odijk T., Macromolecules 12 (1979) 686.

[15] Joosten J.G., McCarthy J.L., Pusey P.N., Macromolecules 24 (1991) 6690.

[16] Doi M. and Edwards S.F., The Theory of Polymer Dynamics (Oxford University Press, 1986).

[17] Brinkman H.C., Appl. Sci. Res. A1 (1947) 27.

[18] Pincus P., Macromolecules 10 (1977) 210.

[19] Joanny J.F., Leibler L., J. Phys. France 51 (1990) 547.

[20] Borue V., Erukhimovich I., Macromolecules 21 (1988) 3240. 